\title{
Neuropsychological function is improved among opioid dependent adults who adhere to opiate agonist treatment with buprenorphine-naloxone: a preliminary study
}

Travis M. Scott ${ }^{1 *}$, Monica Rivera Mindt ${ }^{1}$, Chinazo O. Cunningham² ${ }^{2}$ Franchesca Arias ${ }^{3}$, Kelly Coulehan', Aprille Mangalonzo ${ }^{2}$, Pat Olsen ${ }^{1}$ and Julia H. Arnsten²

\begin{abstract}
Background: Among persons with opioid use disorder (OUD), neuropsychological dysfunction is associated with depression, and better neuropsychological function is associated with opioid abstinence. However, it is unknown whether depressive symptomatology or adherence to opiate agonist treatment are associated with neuropsychological change over time.

Methods: We recruited 20 buprenorphine/naloxone-treated adults with OUD (M Age $=45.2$ years [SD $=8.1] ; 25 \%$ female) to complete baseline and 6 month visits containing a neuropsychological test battery and self-reported measures of depressive symptomatology and medication adherence.
\end{abstract}

Results: Depressive symptomatology was not significantly related to neuropsychological change ( $p$ 's $>.05$ ). Greater adherence to buprenorphine/naloxone was associated with improvements in learning, memory, and global functioning ( $r$ 's = .52-60; $\left.p^{\prime} s<.05\right)$.

Conclusions: Among OUD patients, greater adherence to buprenorphine/naloxone is associated with improved neuropsychological functioning over time. In contrast, depressive symptomatology is not associated with neuropsychological functioning over time. Supporting adherence to buprenorphine/naloxone may improve and/or preserve learning and memory functioning in individuals treated for OUD.

Trial registration: NCT01108679. Registered 21 April 2010.

Keywords: Opioid agonist treatment, Depression, Adherence, Neuropsychological change, Buprenorphine/Naloxone, Opioid use disorder

The most common treatment for opioid use disorder (OUD) is opioid agonist therapy (OAT) with methadone or buprenorphine/naloxone, but little is known about how treatment with buprenorphine/naloxone impacts neuropsychological functioning among persons with OUD. Several factors may affect changes in neuropsychological functioning over time in OUD patients

\footnotetext{
* Correspondence: tshivleyscott@fordham.edu

'Department of Psychology, Fordham University, Bronx, NY 10458, USA

Full list of author information is available at the end of the article
}

receiving treatment with OAT, including depressive symptomatology and medication adherence Exploring these factors in OAT-treated OUD patients might clarify the longitudinal impact of buprenorphine/naloxone treatment among persons at high risk for neuropsychological dysfunction.

\section{OAT and neuropsychological functioning}

In persons with current OUD, neuropsychological impairment is common [1], but data suggest that both methadone and buprenorphine/naloxone improve neuropsychological 
functioning over time in a variety of neuropsychological domains (i.e., verbal learning, visuospatial memory, processing speed, executive functioning) [2, 3]. Improvements in neuropsychological functioning have been identified as early as 2 months after OAT initiation [2]. Preliminary studies indicate that patients taking buprenorphine appear to have better neuropsychological functioning than methadone patients, with medium to large effect sizes in learning and memory, attention/working memory, and executive functioning $[4,5]$. While the results of these studies suggest that buprenorphine/naloxone may improve neuropsychological functioning over time among OUD patients, studies have not examined whether greater medication adherence results in even greater changes in neuropsychological functioning.

\section{Depression and neuropsychological functioning in persons with OUD}

Depression is more prevalent in individuals with OUD than in the general population [6], but little is known about associations between depressive symptomatology and neuropsychological functioning in persons with OUD. Among other substance use disorder populations (e.g., alcohol, cocaine), depressive symptomatology is negatively associated with executive functioning [7], but few studies have examined depression and neuropsychological functioning among persons with OUD specifically. One recent study of opioid-dependent persons found a negative relationship between depressive symptoms and reaction time [8], but more research is needed to understand whether depressive symptomatology attenuates neuropsychological improvement among OUD patients receiving buprenorphine/naloxone treatment.

\section{OAT adherence and neuropsychological functioning}

OAT adherence is typically operationalized as retention in substance use treatment [9], but how OAT retention affects neuropsychological outcomes over time is not well understood. Studies that have collected neuropsychological data from OAT patients have not examined how medication adherence during treatment relates to changes in neuropsychological functioning $[2,3]$. To date, no studies have examined associations between OAT medication-taking adherence and neuropsychological outcomes over time.

\section{Study aims}

We conducted this preliminary study to examine the longitudinal impact of depressive symptomatology and medication adherence on neuropsychological function among OUD patients in treatment with buprenorphine/ naloxone. Our overarching hypothesis was that improvements in neuropsychological functioning over time would occur in: (1) persons with lower depressive symptomatology, and (2) persons with greater medication adherence. We also hypothesized that greater adherence to buprenorphine/naloxone would be independently associated with improved neuropsychological functioning.

\section{Methods \\ Participants}

This preliminary analysis was part of a larger pilot study designed to inform a randomized clinical trial examining neuropsychological effects of methadone and buprenorphine. The pilot study enrolled only participants who were newly initiating buprenorphine/naloxone treatment, who were recruited through word of mouth, flyers, and health care provider referrals.

\section{Inclusion and exclusion criteria}

Inclusion criteria were: 1) current OUD per medical assessment by a physician; 2) no buprenorphine use for 15 consecutive days prior to study enrollment; and 3) anticipated buprenorphine start date within 30 days of study enrollment. Exclusion criteria included: 1) diagnosis of severe psychiatric or medical conditions (e.g., schizophrenia, stroke, head injury with loss of consciousness $>12 \mathrm{~h}$, or seizure disorders); 2) age younger than 18 or older than 65 ; 3) less than 6 years of education; 4) unable to speak English; or 5) not completing all neuropsychological, psychiatric, and adherence measures at the baseline and six-month visits.

\section{Procedures}

Prior to completion of any study evaluations, all participants provided signed informed consent and agreed to complete all neuropsychological, psychiatric, and adherence measures at both the baseline and follow-up visits. After study enrollment, participants underwent buprenorphine/naloxone induction at a community-based clinic which provides integrated substance use disorder and medical care and treatment. After induction, participants were maintained on 4-16 mg of buprenorphine/ naloxone for the six-month duration of the study. Specifically, all participants were in regular medical care and were stable on their buprenorphine/naloxone regimens throughout the study period. Participants received standard-of-care treatment for opioid use disorder and other medical conditions at the same clinic.

Participants completed the measures detailed below at both the baseline and six-month visits. Each visit, including the 90-min neuropsychological test battery, was completed in a single session and took approximately 4 h. Participants were provided with both a snack break and a lunch break during the study visits, and additional breaks as needed. Baseline visits were completed within 14 days of treatment induction, and six-month follow-up 
visits occurred between 165 and 225 days after baseline. The study was approved by the Institutional Review Boards of Albert Einstein College of Medicine and Fordham University.

\section{Measures}

\section{Depressive symptomatology}

At both the baseline and six-month visit, participants completed the Beck Depression Inventory (BDI)-II, which evaluates depressive symptoms over the prior 2 weeks and produces a summary score [10]. We calculated a mean of the two summary scores to estimate depressive symptomatology over the 6 month study period.

\section{Buprenorphine-naloxone adherence}

The Visual Analogue Scale (VAS) measured selfreported adherence over two different 4-week periods, once at the three-month mid-point and once at the sixmonth follow-up visit. At each time point, participants were asked to look at the visual scale and "select the percentage that represents how much of your buprenorphine you've taken in the past 4 weeks." For each participant, we then calculated a mean adherence rate based on their two VAS responses to reflect adherence over the 6 month study period. The VAS is highly correlated with electronic adherence monitoring systems and is well-validated as a measure of antiretroviral adherence $[11,12]$.

\section{Substance use}

Substance use disorder (SUD) diagnoses were determined through computerized structured clinical interviews, the Composite International Diagnostic Interview (CIDI) Version 2.1. Substance use severity was assessed with the Addiction Severity Index (ASI). The CIDI provides diagnostic information based on Diagnostic and Statistical Manual of Mental Disorders (DSM)-IV criteria [13]. The ASI is a structured computerized interview that assesses seven primary domains including alcohol and drug use [14].

\section{Neuropsychological functioning}

At both the baseline and six-month visit, participants completed comprehensive neuropsychological evaluations, including well-validated measures in the following domains: verbal fluency, processing speed, attention/ working memory, learning, memory, motor functioning, and executive functioning (see Table 1 for complete list of neuropsychological measures). Estimated premorbid IQ was assessed with the Wide Range Achievement Test-3 Reading Subtest (WRAT-3). Trained psychometrists administered/scored all batteries and followed standardized procedures. Alternate forms of the learning and memory measures were used for the two visits.
Table 1 Neuropsychological battery and normative data by seven major areas for computation of T-scores and sRCS

\begin{tabular}{ll}
\hline $\begin{array}{l}\text { Neuropsychological Domain } \\
\text { and Test }\end{array}$ & Normative Data Sources [22-27] \\
\hline $\begin{array}{l}\text { Verbal Fluency } \\
\text { Controlled Oral Word Association }\end{array}$ & $\begin{array}{l}\text { Heaton, Miller, Taylor \& Grant } \\
(2004)^{\mathrm{a}, \mathrm{b}, \mathrm{c}, \mathrm{d}}\end{array}$ \\
Test (FAS) & $\begin{array}{l}\text { Heaton, Miller, Taylor \& Grant } \\
(2004)^{\mathrm{a}, \mathrm{b}, \mathrm{c}, \mathrm{d}}\end{array}$ \\
Semantic (Animal) Fluency &
\end{tabular}

Speed of Information Processing

WAIS-III Digit Symbol

WAIS-III Symbol Search

Trail Making Test (Part A)

Attention/Working Memory

WAIS-III Letter Number

Sequencing

PASAT Total Correct

Heaton, Taylor \& Manly $(2003)^{a, b, c, c}$

Heaton, Taylor \& Manly $(2003)^{a, b, c, d}$

Heaton, Miller, Taylor \& Grant $(2004)^{a, b, c, d}$ Heaton, Taylor \& Manly
$(2003)^{a, b, c, d}$

Diehr et al. (2003)

Learning

Hopkins Verbal Learning Test Revised

Benedict, Schretlen, Groninger \& Brandt (1998) ${ }^{a}$

Brief Visuospatial Memory Test - $\quad$ Benedict (1997) ${ }^{a}$ Revised

Memory

Hopkins Verbal Learning Test Revised

Brief Visuospatial Memory Test Revised

Motor

Grooved Pegboard Time

Heaton, Miller, Taylor \& Grant $(2004)^{a, b, c, d}$

Executive Functioning

Wisconsin Card Sorting Kongs, Thompson, Iverson \& Task-64 Item

Trail Making Test (Part B) Heaton (2000) $)^{\mathrm{a}, \mathrm{b}}$

Heaton, Miller, Taylor \& Grant $(2004)^{a, b, c, d}$

Notes. Wechsler Adult Intelligence Scales (WAIS); Paced Auditory Serial Arithmetic Test (PASAT); Summary regression-based change scores (SRCS); Normative data corrects for the demographic characteristics indicated by superscript: ${ }^{\mathrm{a} A g e}{ }^{\mathrm{b}}{ }^{\mathrm{E}}$ education; ${ }^{\mathrm{c}} \mathrm{Gender}$; ${ }^{\mathrm{d}}$ Ethnicity

To assess longitudinal changes in neuropsychological function, summary regression-based change scores (sRCS) were calculated. The sRCS approach accounts for practice effects and utilizes an electronic normative database in which individual $\mathrm{z}$-scores at different time points are computed by dividing the difference between predicted and obtained follow-up scaled scores by the error term of the regression model [15]. The resulting $\mathrm{z}$ score reflects how well or poorly the participant performed at the follow-up time point relative to expectations made from his/her prior score and other variable-specific baseline predictors. The difference 
between the predicted retest performance and actual retest performance on each individual neuropsychological test is then converted into sRCS. For this analysis, sRCS of individual neuropsychological tests from each domain were averaged to create mean domain sRCS, and all individual test sRCS were averaged to create a mean global sRCS. A 90\% confidence interval was used to determine significant global neuropsychological sRCS change, with the top 5\% considered significant improvement and the bottom $5 \%$ considered significant decline.

\section{Statistical analyses}

The independent variables were depressive symptomatology (mean BDI-II summary score over two time points) and adherence to buprenorphine/naloxone (mean adherence rate calculated from two VAS ratings), and the dependent variables were changes in global and domain-specific neuropsychological functioning (global and domain sRCS, with positive scores indicating improvement). We computed Pearson correlations to examine the associations of depressive symptomatology and adherence with global neuropsychological and domain-specific sRCS.

To assess for potential covariates, we examined associations between sRCS and demographic characteristics, Wide Range Achievement Test-3 (WRAT-3) Reading Tscore, urine toxicology, current substance use disorder, days of substance use in past month, and HIV-status. The only significant findings were that gender was related to global and processing speed sRCS (all $p$ 's $<.05$ ), and that WRAT-3 Reading T-score was correlated with global and attention/working memory sRCS (all $p$ 's $<.05)$. Therefore, for all analyses that included the global, processing speed, or attention/working memory sRCS, we computed partial correlations to adjust for gender and WRAT-3 Reading scores.

All analyses were conducted using the Statistical Package for the Social Sciences (SPSS) Version 22.0. Alpha level was set at .05 .

\section{Results}

\section{Participant characteristics}

Table 2 summarizes participants' demographic and clinical characteristics. Participants were mostly male (75\%), middle-aged, and had less than a high school education. Their mean estimated premorbid IQ based on WRAT-3 Reading was in the low average range $(M=86.9, S D=$ 14.0). Most participants reported mild depressive symptomatology, but nearly one-third $(30 \%)$ reported moderate symptoms. Approximately $28 \%$ were $\mathrm{HIV+}$, and mean buprenorphine/naloxone adherence during the six-month study period was $91.3 \%(\mathrm{SD}=11.8)$.

Table 3 summarizes participants' current and past substance use. Sixteen participants had a current substance
Table 2 Participant demographic and selected clinical characteristics $(N=20)$

\begin{tabular}{|c|c|c|}
\hline & $M(S D)$ or $\%(n)$ & Range \\
\hline \multicolumn{3}{|l|}{ Demographic Characteristics } \\
\hline Female & $25 \%(5)$ & \\
\hline \multicolumn{3}{|l|}{ Race/Ethnicity } \\
\hline Hispanic/Latina/o & $55 \%(11)$ & \\
\hline African American & $25 \%(5)$ & \\
\hline Other/Not listed & $15 \%(3)$ & \\
\hline Non-Hispanic white & $5 \%(1)$ & \\
\hline Age & $45.2(8.1)$ & $32-61$ \\
\hline Years of Education & $11.7(2.3)$ & $7-18$ \\
\hline WRAT-3 Reading Subtest Standard Score ${ }^{a}$ & $86.9(14.0)$ & $66-109$ \\
\hline HIV-Seropositive & $28 \%(5)$ & \\
\hline \multicolumn{3}{|l|}{ Depressive Symptomatology } \\
\hline BDI-II Total Score & $13.2(9.2)$ & $0-29.5$ \\
\hline$\%$ Adherence $^{c}$ & $91.4(11.8)$ & $64-100$ \\
\hline \multicolumn{3}{|c|}{$\begin{array}{l}\text { Notes } \\
\text { aWRAT-3 Reading = Wide Range Achievement Test-3 Reading Subtest standard } \\
\text { score from baseline visit } \\
\text { bBDI-II Total Score }=\text { mean Beck Depression Inventory-II Total Score from base- } \\
\text { line and } 6 \text { month visit } \\
\text { cAdherence }=\% \text { mean adherence from visual analogue scale at two points } \\
\text { (midpoint and six-month follow up visit) }\end{array}$} \\
\hline
\end{tabular}

use disorder (SUD) according to the CIDI, but all 20 had a lifetime SUD diagnosis. At baseline, 14 participants (70\%) had a urine toxicology test that revealed substance use (i.e., amphetamines, benzodiazepines, cocaine, opiates, methadone, and/or oxycodone), and at the six-month follow-up visit 13 participants (65\%) had a positive urine toxicology result. Three participants had negative urine toxicology results at both time points. Participants self-reported using heroin for a mean of 16.4 years $(S D=12.8)$, using methadone for a mean of 3.9 years $(S D=5.1)$, and using other opiates (e.g., opioid analgesics) for a mean of 4.2 years $(S D=8.1)$. In the month prior to the baseline evaluation, heroin was the most frequently used substance, with mean usage of 8.6 days $(S D=10.2)$. In the month prior to the six-month follow-up visit, other opiates were the most frequently used substance, with mean usage of 1.8 days $(S D=5.8)$.

Table 4 summarizes participants' neuropsychological (NP) characteristics. Over the course of 6 months, 15\% of participants showed global NP improvement, $5 \%$ had global NP decline, and $80 \%$ remained stable. The mean global sRCS for the sample was -.01 $(S D=.46)$, indicating no significant overall NP change. Changes in specific $\mathrm{NP}$ domains ranged from a mean sRCS of $-.22(S D=.50)$ in learning to a mean sRCS of $.41(S D=.92)$ in executive functioning, indicating minimal change in domain-specific NP functioning. 
Table 3 Participant current and past substance use characteristics $(N=20)$

\begin{tabular}{|c|c|}
\hline & $M(S D)$ or $\%(n)$ \\
\hline \multicolumn{2}{|c|}{ CIDI Lifetime Substance Use Disorder } \\
\hline Cocaine & $70 \%(14)$ \\
\hline Alcohol & $60 \%(12)$ \\
\hline Cannabis & $50 \%(10)$ \\
\hline Hallucinogens & $15 \%(3)$ \\
\hline Sedatives & $15 \%(3)$ \\
\hline \multicolumn{2}{|c|}{ Positive Urine Toxicology Result (Baseline visit) } \\
\hline Opiates & $35 \%(7)$ \\
\hline Cocaine & $35 \%(7)$ \\
\hline Methadone & $20 \%(4)$ \\
\hline Benzodiazepines & $10 \%(2)$ \\
\hline Oxycodone & $10 \%(2)$ \\
\hline \multicolumn{2}{|c|}{ Positive Urine Toxicology Result (6 Month visit) } \\
\hline Opiates & $42 \%(8)$ \\
\hline Cocaine & $31 \%(6)$ \\
\hline Methadone & $15 \%(3)$ \\
\hline Amphetamines & $11 \%(2)$ \\
\hline \multicolumn{2}{|c|}{ ASI Days of Substance Use in Prior Month (Baseline) } \\
\hline Alcohol Intoxication & $3.2(6.8)$ \\
\hline Heroin & $8.6(10.2)$ \\
\hline Methadone & $2.3(5.8)$ \\
\hline Other Opiates & $2.6(5.5)$ \\
\hline Benzodiazepines & $0.5(1.6)$ \\
\hline Cocaine & $3.5(6.9)$ \\
\hline \multicolumn{2}{|c|}{ ASI Days of Substance Use in Prior Month (6 Month visit) } \\
\hline Alcohol Intoxication & $1.3(1.9)$ \\
\hline Heroin & $1.6(3.2)$ \\
\hline Methadone & $0.1(0.2)$ \\
\hline Other Opiates & $1.8(5.8)$ \\
\hline Benzodiazepines & $0.4(1.6)$ \\
\hline Cocaine & $1.2(3.4)$ \\
\hline
\end{tabular}

Notes. CIDI Composite International Diagnostic Interview, ASI Addiction Severity Index

\section{Depression, adherence, and neuropsychological functioning}

Depressive symptomatology (mean BDI-II score) was not significantly correlated with global NP change (sRCS) or NP change in any NP domains (all $r$ 's $=-.02$ to -.41 ; all $p$ 's $>.05)$, nor was it significantly correlated with adherence $(r=-.05 ; p=.84)$. The results of remaining correlational analyses revealed that greater adherence to buprenorphine/naloxone was associated with improved learning sRCS $(r=.52, p=.019)$, memory $\operatorname{sRCS}(r=.59, p=.006)$, and global sRCS $(r=.60, p$ $=.008)$, and these effects were in the medium range [16].
Table 4 Participant neuropsychological (NP) characteristics at baseline and follow-up ( $N=20)$

\begin{tabular}{llll}
\hline & Time 1 & Time 2 & sRCS \\
& T-score M(SD) & T-score M(SD) & M(SD) \\
\hline NP Domains & & & \\
Global & $41.8(6.4)$ & $44.5(6.2)$ & $-.01(.46)$ \\
Learning & $36.2(11.7)$ & $37.8(9.3)$ & $-.22(.50)$ \\
Memory & $36.9(12.2)$ & $38.5(11.4)$ & $-.12(.89)$ \\
Verbal Fluency & $46.3(9.0)$ & $47.3(12.0)$ & $-.13(.94)$ \\
Processing Speed & $49.5(8.6)$ & $52.2(8.8)$ & $-.09(.88)$ \\
Attention/Working Memory & $44.3(8.0)$ & $46.9(9.2)$ & $.04(.98)$ \\
Motor & $39.2(9.9)$ & $42.9(9.0)$ & $.09(.68)$ \\
Executive Functioning & $41.7(6.7)$ & $46.2(8.8)$ & $.41(.92)$ \\
\hline Notes. sRCS summary regression-based change scores
\end{tabular}

\section{Discussion}

We found that self-reported adherence to buprenorphine/naloxone treatment over time is high, and that greater adherence is associated with improvement in learning and memory neuropsychological functioning. Although we found that adherence is also significantly related to improved global neuropsychological functioning, this finding was likely driven by the significant positive associations of adherence with learning and memory. By measuring OAT adherence over time and as a continuous variable, we have identified the unique impact of greater adherence on the neuropsychological domains of learning and memory.

Our finding that greater medication adherence is associated with improved neuropsychological functioning (i.e., learning and memory) over the course of 6 months in OUD patients is consistent with previous research $[17,18]$. For example, one review article concluded that neuropsychological impairment is related to involuntarily dropping out of substance abuse treatment, and that duration of abstinence is correlated with improvement in neuropsychological functioning [18]. Similar to ours, another study found that patients receiving substance use disorder treatment (i.e., in a chemical dependency treatment program or in residential or intensive day treatment) have the greatest neuropsychological improvement over 6 weeks in the area of memory [17]. In contrast to prior studies $[2,3,5]$, our study did not find a significant relationship between adherence to buprenorphine/naloxone and improved attention/working memory, processing speed, or executive functioning. However, prior studies did not examine the relationship between adherence and other domains of neuropsychological functioning, which highlights the specificity of our finding that adherence to buprenorphine/naloxone was uniquely associated with learning and memory, but not with other neuropsychological domains. This 
represents a highly novel finding for the field that warrants further study. Our study also extends prior findings by defining treatment adherence beyond program attendance, and by examining both adherence to medication-taking and neuropsychological functioning over a significantly longer period. Additionally, other studies have not explored the impact of other important variables, such as depression, on neuropsychological functioning over time.

Unlike prior studies $[7,8,19]$, we did not find significant associations between depressive symptomatology and neuropsychological change over time, despite nearly one-third of the sample reporting moderate depressive symptomatology. However, the prior cross-sectional study by Beatty et al. [7] was conducted within a sample of patients with alcohol and cocaine use disorders, not patients with OUD. Another prior study by Horner et al. [19] did not use objective tests of neuropsychological function, instead measuring neuropsychological functioning through self-evaluation in a broadly defined sample of substance use disorder patients. A previous study by Loeber et al. [8] reported a significant association between depression and neuropsychological functioning in OUD patients using objective neuropsychological tests, but this foreign study had methodological differences from ours (e.g., longitudinal design, different neuropsychological measures). In addition, a review article concluded that the association between depression and neuropsychological functioning in patients with substance use disorders is equivocal [18].

Our study has several important implications. First, it advances understanding of neuropsychological outcomes among persons with OUD by quantifying OAT adherence rather than measuring attendance in a treatment program [17]. We have thus identified a potential target (medication-taking) to intervene to improve neuropsychological outcomes in a population with high rates of cognitive impairment. Second, unlike most prior studies, our longitudinal design enhances the predictive validity of our findings. Third, we used well-validated measures of depressive symptomatology and neuropsychological function, addressing limitations with prior research (e.g., Horner et al. [19]). Fourth and finally, the inclusion of a U.S.-based, ethnically diverse sample permits generalizability to those most at risk for depressive symptomatology and SUD. Though prior research suggests that ethnically diverse individuals have higher rates of mild and moderate depression than non-Hispanic whites [20] and are overrepresented among those with OUD in low-income areas [21], there is little research on the impact of OAT and depressive symptomatology on neuropsychological functioning in this population.

Despite its strengths, our study has limitations. The racial/ethnic diversity of our sample is both a strength and a limitation, as it is unknown whether our findings are generalizable to a predominantly non-Hispanic white sample. While this study provided a comprehensive overview of participants' current substance use, an additional limitation was that information about other prescribed medications (unrelated to OAT) was not available. Because we did not have a non-treated control group, our conclusions are limited regarding how study participants compare to OUD patients not receiving treatment with buprenorphine/naloxone. However, our analyses accounted for many demographic characteristics known to impact neuropsychological test performance. Because this is a preliminary study, these findings should inform future, larger-scale studies in which non-treated control groups could be added. Considering that eight correlational analyses were performed, the .05 alpha level used in the present study is a limitation. However, applying an alpha correction, such as Bonferroni, would have significantly reduced the power given the limited sample size. Finally, medication adherence was only assessed through self-report. Given that there is no 'gold standard' for adherence to buprenorphinenaloxone, future studies would benefit from multiple measures of adherence to better contextualize the current findings.

Future studies should replicate these findings with a larger and more diverse sample. Additionally, research should explore the relationship between buprenorphine/ naloxone treatment and other specific areas of neuropsychological functioning (e.g., reaction time) that have been associated with depression in OUD patients (e.g., Loeber et al. [8]) Lastly, studies should continue to explore the relationship between medication-taking adherence and neuropsychological functioning over time, using different medications and/or different ways of measuring adherence. For example, future longitudinal studies might use objective measures of adherence to examine the relationship between OAT adherence and neuropsychological functioning, or might test interventions, such as directly observed therapy, to enhance adherence.

\section{Conclusions}

In sum, we found that greater adherence to buprenorphine/naloxone treatment is associated with improved learning and memory over time among persons with OUD. This finding illustrates the importance of medication adherence in improving neuropsychological functioning within this population. Supporting OAT adherence may be a particularly important component of improving and/or preserving learning and memory functioning in individuals treated for OUD.

\section{Acknowledgements}

The authors acknowledge Zohar Massey, Mia Brisbane, Katie Segal, Yuming Ning, Felicia Fraser, and Xia Ha for expert technical assistance, and also the patients who generously gave their time to this study. 


\section{Funding}

This work was supported by National Institutes of Health [R01DA032552 to $M R M \& J A]$.

\section{Availability of data and materials}

The datasets used and/or analyzed are available from the corresponding author on reasonable request.

\section{Authors' contributions}

All authors contributed to the preparation of this manuscript. MRM, CC, and JA contributed to study design and implementation. Data collection was performed by FA and KC. Data analysis was performed by TS and MRM. All authors read and approved the final manuscript.

\section{Ethics approval and consent to participate}

Informed consent was gathered for all participants with the approval from the Institutional Review Boards of Albert Einstein College of Medicine and Fordham University.

\section{Consent for publication}

Not applicable.

\section{Competing interests}

The authors declare that they have no competing interests.

\section{Publisher's Note}

Springer Nature remains neutral with regard to jurisdictional claims in published maps and institutional affiliations.

\section{Author details}

'Department of Psychology, Fordham University, Bronx, NY 10458, USA.

${ }^{2}$ Department of Medicine, Albert Einstein College of Medicine and Montefiore Medical Center, Bronx, NY 10467, USA. ${ }^{3}$ Department of Psychology, University of Florida, Gainesville, FL 32611, USA.

Received: 4 July 2017 Accepted: 7 November 2017

Published online: 15 November 2017

\section{References}

1. Baldacchino A, Balfour DJK, Passetti F, Humphris G, Matthews K. Neuropsychological consequences of chronic opioid use: a quantitative review and meta-analysis. Neurosci Biobehav Rev. 2012;36(9):2056-68. doi:10.1016/j.neubiorev.2012.06.006.

2. Gruber SA, Tzilos GK, Silveri MM, et al. Methadone maintenance improves cognitive performance after two months of treatment. Exp Clin Psychopharmacol. 2006;14(2):157-64. doi:10.1037/1064-1297.14.2.157.

3. Singhal A, Tripathi BM, Pal HR, Jena R, Jain R. Effect of buprenorphine on psychomotor functions in patients on buprenorphine maintenance. J Opioid Manag. 2007:4(1):41-7.

4. Pirastu $R$, Fais $R$, Messina $M$, et al. Impaired decision-making in opiatedependent subjects: effect of pharmacological therapies. Drug Alcoho Depend. 2006;83(2):163-8. doi:10.1016/j.drugalcdep.2005.11.008.

5. Rapeli P, Fabritius C, Alho H, Salaspuro M, Wahlbeck K, Kalska H. Methadone vs. buprenorphine/naloxone during early opioid substitution treatment: a naturalistic comparison of cognitive performance relative to healthy controls. BMC Clin Pharmacol. 2007;7(1):5. doi:10.1186/1472-6904-7-5.

6. Goldner EM, Lusted A, Roerecke M, Rehm J, Fischer B. Prevalence of Axis-1 psychiatric (with focus on depression and anxiety) disorder and symptomatology among non-medical prescription opioid users in substance use treatment: systematic review and meta-analyses. Addict Behav. 2014:39(3):520-31. doi:10.1016/j.addbeh.2013.11.022

7. Beatty WW, Katzung VM, Moreland VJ, Nixon SJ. Neuropsychological performance of recently abstinent alcoholics and cocaine abusers. Drug Alcohol Depend. 1995:37(3):247-53. doi:10.1016/0376-8716(94)01072-S.

8. Loeber S, Nakovics H, Kniest A, Kiefer F, Mann K, Croissant B. Factors affecting cognitive function of opiate-dependent patients. Drug Alcohol Depend. 2012;120(1-3):81-7. doi:10.1016/j.drugalcdep.2011.07.001.

9. Connock M, Juarez-Garcia A, Jowett $\mathrm{S}$, et al. Methadone and buprenorphine for the management of opioid dependence: a systematic review and economic evaluation, NIHR Health Technology Assessment Programme: executive summaries. Southampton (UK): NIHR Journals Library; 2007.
10. Beck AT, Steer RA, Gk B. BDI-II, Beck depression inventory: manual. San Antonio, TX: Psychological Corporation; 1996.

11. Shi L, Liu J, Koleva Y, Fonseca V, Kalsekar A, Pawaskar M. Concordance of adherence measurement using self-reported adherence questionnaires and medication monitoring devices. PharmacoEconomics. 2010;28(12):1097-107. doi:10.2165/11537400-000000000-00000

12. Simoni JM, Kurth AE, Pearson CR, Merrill JO, Frick PA. Self-report measures of antiretroviral therapy adherence: A review with recommendations for HIV research and clinical management. 2014;10(3):227-245. doi:10.1007/s10461006-9078-6

13. World Health Organization. CIDI-auto version 2.1: Administrator's guide and reference. Sydney, Australia: Training and reference centre for WHO CIDI; 1997.

14. McLellan AT, Kushner $H$, Metzger $D$, et al. The fifth edition of the addiction severity index. J Subst Abus Treat. 1992;9(3):199-213. doi:10.1016/07405472(92)90062-S.

15. Cysique LA, Franklin D, Abramson I, et al. Normative data and validation of a regression based summary score for assessing meaningful neuropsychological change. J Clin Exp Neuropsychol. 2011;33(5):505-22. doi:10.1080/13803395.2010.535504.

16. Murphy KR, Myors B, Wolach A. Statistical Power Analysis: A Simple and General Model for Traditional and Modern Hypothesis Tests. New York, NY: Routledge; 2014

17. Bates ME, Voelbel GT, Buckman JF, Labouvie EW, Barry D. Short-term neuropsychological recovery in clients with substance use disorders. Alcohol Clin Exp Res. 2005;29(3):367-77. doi:10.1097/01.ALC.0000156131.88125.2A.

18. Verdejo-García A, López-Torrecillas F, Giménez CO, Pérez-García M. Clinical implications and methodological challenges in the study of the neuropsychological correlates of cannabis, stimulant, and opioid abuse. Neuropsychol Rev. 2004;14(1):1-41.

19. Horner MD, Harvey RT, Denier CA. Self-report and objective measures of cognitive deficit in patients entering substance abuse treatment. Psychiatry Res. 1999:86(2):155-61. doi:10.1016/S0165-1781(99)00031-1.

20. Pratt LA, Brody DJ. Depression in the U.S. household population, 2009-2012. NCHS data brief, no. 172. Hyattsville, MD: National Center for Health Statistics; 2014

21. American Psychiatric Association. Diagnostic and Statistical Manual of Mental Disorders (DSM-5). Washington, DC: American Psychiatric Pub; 2013.

22. Heaton RK, Miller SW, Taylor MJ, Grant I. Revised comprehensive norms for an expanded Halstead-Reitan Battery: demographically adjusted neuropsychological norms for African American and Caucasian adults. Lutz: FL Psychol Assess Resour; 2004.

23. Heaton RK, Taylor MJ, Manly J. Demographic Effects and Use of Demographically Corrected Norms with the WAIS-III and WMS-III. In: Tulsky DS, Saklofske DH, Chelune GJ, Heaton RK, Ivnik RJ, Bornstein R, Prifitera A, \& Ledbetter MF, eds. Clinical interpretation of the WAIS-III and WMS-III. San Diego: Academic Press; 2003.

24. Diehr MC, Cherner M, Wolfson TJ, Miller SW, Grant I, Heaton RK. The 50 and 100-item short forms of the Paced Auditory Serial Addition Task (PASAT): demographically corrected norms and comparisons with the full PASAT in normal and clinical samples. J Clin Exp Neuropsychol. 2003;25(4):571-85. doi:10.1076/jcen.25.4.571.13876.

25. Benedict RHB, Schretlen D, Groninger L, Brandt J. Hopkins verbal learning test-revised: normative data and analysis of inter-form and test-retest reliability. Clin Neuropsychol. 1998;12(1):43-55.

26. Benedict RHB. Brief visuospatial memory test-revised: Professional Manual. Lutz, FL: PAR; 1997.

27. Kongs SK, Thompson LL, Iverson GL, Heaton RK. Wisconsin card sorting Test-64 card version (WCST-64). Odessa: FL Psychol Assess Resour; 2000.

\section{Submit your next manuscript to BioMed Central} and we will help you at every step:

- We accept pre-submission inquiries

- Our selector tool helps you to find the most relevant journal

- We provide round the clock customer support

- Convenient online submission

- Thorough peer review

- Inclusion in PubMed and all major indexing services

- Maximum visibility for your research

Submit your manuscript at www.biomedcentral.com/submit
Biomed Central 